\title{
Globalisierung und translokales Kulturverständnis
}

\section{Norman Backhaus und Marco Hoffmann, Zürich}

\section{Einleitung: Globalisierung als Diskurs}

Der Begriff Globalisierung ist geradezu zum Un- oder Schreckenswort des ausgehenden Jahrhunderts geworden (Beck 1997: 1, Schmidt 1998). Die Globalisierung wird ebenso für Klimaveränderungen und damit in Zusammenhang gestellte vergrösserte Risiken von Naturgefahren verantwortlich gemacht, wie für soziale Veränderungen, die mit Arbeitsplatzvernichtung oder Identitätsverlust (GOLLMER 1999, WAGNER 1996) umschrieben werden. Sie wird so gleichsam zur Akteurin gemacht, der man ausgeliefert ist und die unser Alltagsleben bedroht, wie dies z.B. Hans KaUfManN, Exökonom der Bank Julius Bär ausdrückte: «Man rief die Globalisierung, und jetzt wird man ihre Folgen nicht mehr los» (SPIElER 1998). Es gibt aber auch Stimmen, welche die Folgen von Globalisierung positiv beurteilen und sie mit Egalisierung und Demokratisierung verbinden (ARNASON 1990, Panajotis 1996), und davon ausgehen, dass durch die Universalisierung bspw. der Menschenrechte, Spannungen und Konfliktpotentiale abgebaut werden können.

In diesem Artikel soll die These dargelegt und verteidigt werden, dass das Verständnis von Kultur die Wahrnehmung der Globalisierung - und damit verbunden, den Diskurs darüber - bestimmt. Versteht man Globalisierung einerseits einseitig als homogenisierend und verbindet man dies anderseits mit einem Kulturverständnis, das Kultur als räumlich und zeitlich statisch sieht wie dies in der Geographie häufig der Fall ist -, so darf es nicht überraschen, dass Globalisierung, mit der viele (fremde) Kulturelemente transportiert werden (z.B. durch Medien, Migrationen, Konsum, Produktion, Kapitalverschiebungen etc.), von vielen als Bedrohung empfunden wird. Wir möchten in unserem Beitrag ein weitergefasstes Globalisierungskonzept vorstellen, das homogenisierende mit fragmentierenden Prozessen verknüpfen kann sowie ein nicht-statisches, fliessendes und translokales Kulturverständnis entwerfen. Es ist unseres Erachtens am ehesten dazu geeignet, für die gesellschaftlichen Probleme - wie z.B. die unter dem Begriff «global change» zusammengefassten Veränderungen, die Vergrösserung von sozialen Disparitäten oder Verunsicherungen über beschleunigte Veränderungen von Werten und Normen - unter Bedingungen der Globalisierung Lösungsansätze zu finden.

Die Wahrnehmung und Bewertung der (kulturellen) Globalisierung beruht gemäss dieser Argumentation darauf, wie Kultur und Globalisierung vorgestellt und diskutiert werden. In diesem Artikel sollen demzufolge nicht Globalisierung und Kultur problematisiert werden, sondern Konzeptionen von Globalisierung und Kultur sowie ihre Implikationen und Konsequenzen.

Durchsucht man Zeitungen und Zeitschriften nach dem Begriff «Globalisierung», so wird man mit zahlreichen «Treffern» belohnt (HofFmanN 1999). Dies ist ein klares Indiz dafür, dass in der Öffentlichkeit ein reger Diskurs über dieses Phänomen geführt wird. Darüber hinaus vergeht kaum eine Woche, in der der Begriff nicht den Titel einer wissenschaftlichen Neuerscheinung ziert. Im Folgenden soll dieser Globalisierungsdiskurs (vgl. Abbildung) kritisch betrachtet werden. In seinen Dimensionen manifestieren sich gesellschaftliche Konflikte und Sichtweisen, die über den Begriff «Globalisierung» in einen neuen Zusammenhang gestellt werden. Der Begriff ist erst seit relativ kurzer Zeit ein Element des gesellschaftlichen Diskursgeflechts - er tauchte 1962 erstmals im Websters Dictionary auf (ROBERTSON 1992) -, doch beginnt damit nicht unvermittelt ein neuer, unabhängiger Diskursstrang, sondern er knüpft an andere, bestehende Diskurse an, nimmt deren Themen teilweise auf, verbindet und bündelt sie auf neue Weise. Dabei strukturiert die weltanschauliche Haltung die Wahrnehmung und die Interpretation der Globalisierung. So werden darin bspw. Verbindungen zwischen fortschreitender weltwirtschaftlicher Integration und einer Neuorientierung der nationalen Wirtschaftspolitik oder zwischen der Verbreitung us-amerikanischer Populärkultur und der Bedrohung der kulturellen Identität hergestellt. Man kann den Globalisierungsdiskurs auch als Diskurs über die Definition einer sich im Fluss befindlichen Welt verstehen. Somit wird klar, dass er weder auf Propaganda oder Ideologie, noch auf einen Modediskurs reduziert werden kann; es geht um weit mehr: Es geht um eine gesellschaftliche Orientierung in einer sich verändernden Welt.

Der öffentliche Diskurs wird auf verschiedenen Ebenen geführt. Eine zentrale Rolle nimmt der Mediendiskurs ein, da moderne Öffentlichkeit weitgehend medial hergestellt wird. Auch der Wissenschaftsdiskurs wird, wenn auch oft nur in speziellen Foren und Publikationen, zu einem grossen Teil öffentlich gemacht. In diesem Artikel werden stellvertretend für den gesamten öffentlichen Diskurs, der Medien- und der Wissenschaftsdiskurs betrachtet. Sie können einander gegenübergestellt werden, wobei anzumerken ist, dass sich die beiden Diskursstränge überlappen und gegenseitig beeinflussen. Werden im medialen Diskurs v.a. wirtschaftliche Aspekte der Globalisierung zum Thema macht, ist der wissenschaftliche Diskurs weiter gefächert. In den folgenden Abschnitten sollen zunächst die 


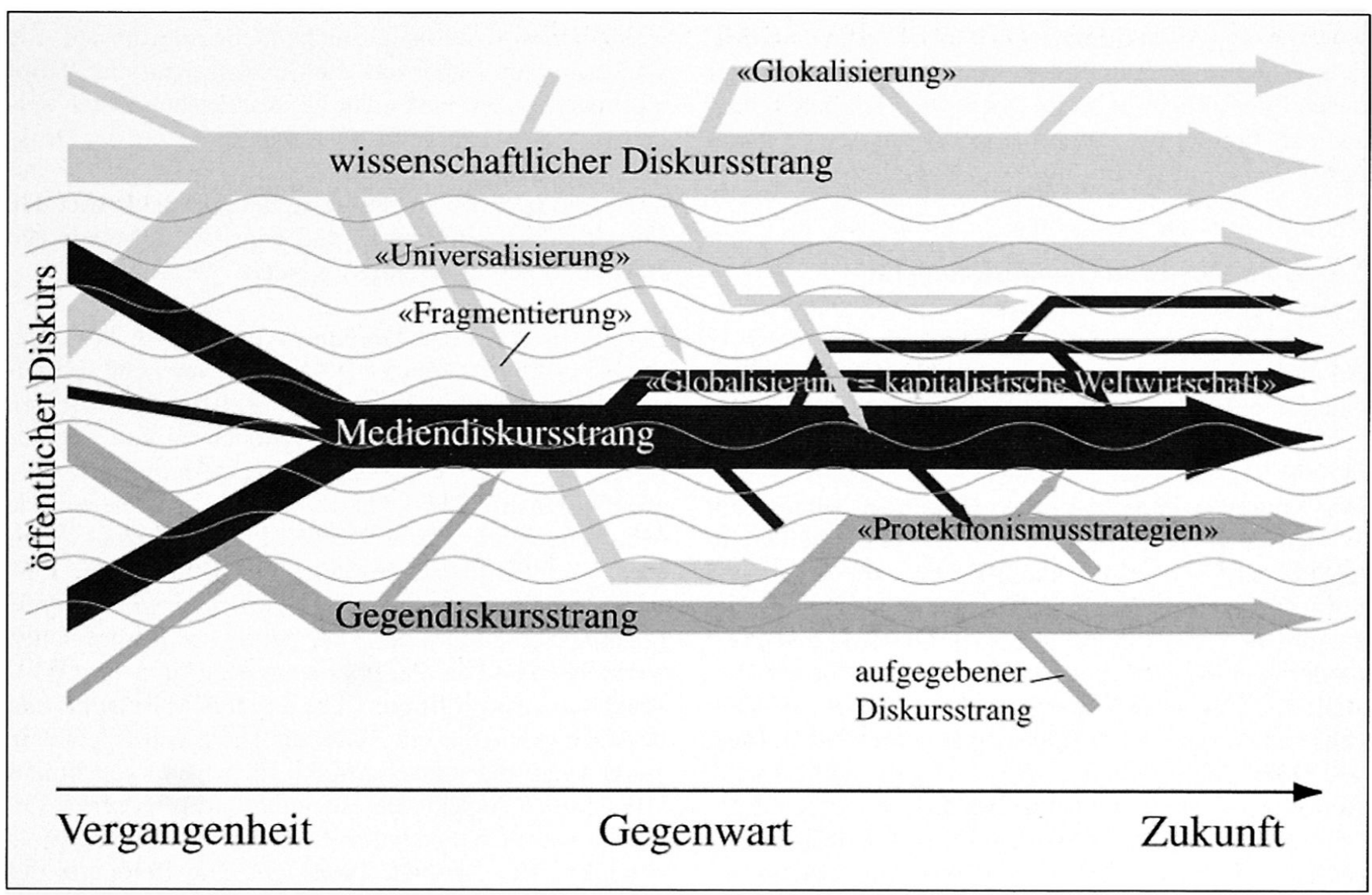

Abb. 1: Modell des Diskursgeflechts über Globalisierung Model of the network of discourses about globalization Modèle du résau des discours sur la mondialisation

wichtigsten Tendenzen im Mediendiskurs aufgezeigt und danach verschiedene wissenschaftliche Konzeptionen von Globalisierung mit den damit verbundenen Prozessen diskutiert werden.

\section{Globalisierung im Mediendiskurs}

Wie ein Begriff in der Gesellschaft wahrgenommen wird, hängt stark von seiner Repräsentation in den Medien ab. In einer Inhaltsanalyse zweier führender Schweizer Tageszeitungen - Neue Zürcher Zeitung und Tages-Anzeiger - wurden Bedeutungen und Konnotationen des Begriffs «Globalisierung» untersucht (Hoffmann 1999). Die Ergebnisse zeigen, dass er am häufigsten als Synonym für das wirtschaftliche Zusammenwachsen der Welt verwendet wurde, das sich durch geographische Ausweitung von Wirtschaftsaktivitäten und durch die Zunahme der Grösse und Geschwindigkeit von Kapital- und Handelsströmen auszeichnet. Allerdings wird Globalisierung hier oft verdinglicht und als etwas Unabwendbares, Äusseres verstanden, dem man nicht entgehen kann. Häufig hat sie eine negative Konnotation oder wird als Sachzwang mit negativen
Auswirkungen angesehen, wie es bspw. erhöhter Wettbewerbsdruck und damit verbunden der Zwang zu Rationalisierungen und schliesslich Entlassungen darstellen. Doch wird Globalisierung auch als Chance verstanden, neue Wege zu gehen, Nischen zu entdecken und neue Märkte zu erschliessen. Als Konsequenz aus der Wahrnehmung von Globalisierung als ökonomischem Sachzwang ergeben sich Forderungen nach staatlichen Strukturen, die sich an die neuen, sich rascher ändernden Rahmenbedingungen der Globalisierung anpassen sollen. Gleichzeitig wird moniert, dass Nationalstaaten und Parteien überfordert seien und an Einfluss verlören. Die Forderung der Stärkung schon bestehender oder neu zu schaffender supranationaler Organisationen, um ein Gegengewicht zur Globalisierung der Wirtschaft herzustellen, ist ebenfalls ein wichtiges Thema im öffentlichen Diskurs. Es werden jedoch auch Stimmen laut, welche die Gesellschaft mit protektionistischen Massnahmen vor den als negativ betrachteten Auswirkungen der Globalisierung schützen wollen. Schliesslich - allerdings weit weniger häufig - wird Globalisierung auch als Signatur unserer Epoche verstanden, die verwendet wird, um die gegenwärtigen Wandlungstendenzen allgemein zu benennen. 
Auch im wissenschaftlichen Diskurs treten unterschiedliche Konzeptionen und Bewertungen auf, die mitunter ebenso pessimistisch sind. Doch wird Globalisierung auch als Formel für eine Neuausrichtung des Verständnisses von Gesellschaft und Kultur beschrieben.

\section{Konzeptionen von Globalisierung im wissenschaftlichen Diskurs}

An dieser Stelle kann nicht der gesamte wissenschaftliche Globalisierungsdiskurs wiedergeben werden, zu zahlreich ist die Literatur, die in den letzten Jahren zu diesem Thema erschienen ist (vgl. BackHaus 1999). Das zeigt allerdings, dass dem Phänomen auch in der Wissenschaft grosse Beachtung geschenkt wird. Die Intensität der Diskussion veranlasste FEATHERSTONE und LASH (1995: 3) gar von «Globalisierung als Paradigma» zu sprechen, das die Debatte über Moderne und Postmoderne ablöst und eine voll entwickelte Theorie darstellt. Im Folgenden sollen stellvertretend für eine Vielzahl von Ansätzen drei Richtungen vorgestellt werden, wie Globalisierung in der Wissenschaft diskutiert wird. Ausgehend von einer Denkweise, die die Wirtschaft ins Zentrum stellt (z.B. von WALLERSTEIN), wird anschliessend eine Position erläutert, die die Mehrdimensionalität der Globalisierung betont (z.B. von GidDENS). Schliesslich wird ein Konzept erläutert, das die bisherigen Dimensionen um eine Perspektive ergänzt, welche die zentrale Bedeutung der Kultur für das Verständnis der Globalisierung hervorhebt (z.B. von ROBERTSON).

\subsection{Globalisierung oder} wirtschaftliche Internationalisierung?

Die Ausbreitung des kapitalistischen Weltmarkts ist eine der wichtigsten und bestimmendsten Prozesse in unserem Alltag, wenngleich nicht der einzige, wie noch zu zeigen sein wird. Wir nehmen dies dadurch wahr, dass wir Produkte, die weit entfernt von uns produziert werden, konsumieren können, aber auch durch die «Vernichtung» von Arbeitsplätzen aufgrund von Produktionsverlagerungen und Rationalisierungen. Oft wird die Frage gestellt, ob wir es hier wirklich mit Globalisierung oder «nur» mit einer verstärkten Internationalisierung der Wirtschaft zu tun haben. SkeptikerInnen wie Gordon, Glyn \& Sutcliffe, Hirst \& Thompson (Dicken 1998: 3, Perraton et al. 1998: 135) bezweifeln die Neuheit der gegenwärtigen Situation, da die Weltökonomie bereits vor dem Ersten Weltkrieg ebenso integriert gewesen sei wie heute. Ihnen kann entgegengehalten werden, dass sie sich v.a. auf die Handelstätigkeit abstützen und den Produktionsprozess, der sich in erster Linie verlagert und globalisiert hat, vernachlässigen. Die «Hyper-GlobalisierungstheoretikerInnen» wie Ohmae und Reich (Dicken 1998: 3, Giddens 1999: 40, Perraton et al. 1998: 135) postulieren dagegen, dass wir bereits in einer Welt ohne Grenzen lebten, in welcher das «Nationale» nicht mehr relevant sei. Sie schiessen damit über das Ziel hinaus, denn das Nationalstaatensystem darf nicht als obsolet betrachtet werden, da viele wichtige Entscheidungen - trotz des Drukkes transnationaler Unternehmen - noch immer auf internationaler Ebene ausgehandelt, in multilateralen Verträgen festgeschrieben und von nationalstaatlichen Institutionen implementiert werden.

Bereits in den 70er Jahren hat ImManuel WallerSTEIN (1990, ROBERTSON 1992: 65ff.) versucht, mit seiner Weltsystemtheorie, die globalen wirtschaftlichen Zusammenhänge und Abhängigkeiten zu konzeptualisieren. Mit seiner vielbeachteten Einteilung in Kernregion (Industrieländer, bzw. Triade), Semiperipherie (Schwellenländer, bzw. Newly Industrializing Countries) und Peripherie (Entwicklungsländer, bzw. Low und Least Developed Countries) drückt er globale Ausbeutungsverhältnisse aus, bei denen die Kernregionen am meisten und die Peripherie am wenigsten vom Wirtschaftsprozess profitieren. Die auf dem Marxismus und der Dependenztheorie fussende Theorie betrachtet in erster Linie die wirtschaftliche Ebene und subsumiert alle anderen Aspekte des sozialen und politischen Lebens - also auch die Nationalstaaten - unter dem Primat der Ökonomie (BEyER 1994: 21). Die Betonung des Wirtschaftlichen als Hauptprozess der Globalisierung findet sich besonders im öffentlichen Diskurs wieder, wenngleich die «ausbeuterische» Haltung der Kernregion heute weniger im Zentrum steht als noch vor zehn, fünfzehn Jahren.

\subsection{Mehrdimensionalität und die Umstrukturierung von Zeit und Distanz}

Die eindimensionale Fokussierung auf wirtschaftliche Prozesse wird dem Phänomen Globalisierung nicht gerecht, auch wenn der öffentliche Diskurs v.a. von dieser Dimension beherrscht wird. ANTHONY GIDDENS (1992, 1999) lehnt es z.B. ab, Globalisierung nur - oder auch nur in erster Linie - ökonomisch zu betrachten, auch wenn die wirtschaftliche Dimension sehr wichtig ist. Im Gegensatz zu WALLERSTEIN nähert er sich dem Phänomen nicht über die Analyse von (Welt-)Systemen, sondern - im Einklang mit seiner Theorie der Strukturierung (vgl. GidDENS 1995) - über die Handlungen der einzelnen Individuen, die dadurch zum gestaltenden Teil des Prozesses werden und nicht passive Opfer eines auf sie einwirkenden Systems sind.

Globalisierung soll primär als Umstrukturierung von Zeit und Distanz im sozialen Leben verstanden werden. In traditionellen Gesellschaften dominierten face-toface Kontakte, also direkte Begegnungen von Menschen, und die Konsequenzen einer Handlung waren i.d.R. zeitlich und räumlich in der Nähe ihres Ausgangspunktes lokalisierbar. In post-traditionellen, modernen, spät-modernen oder postmodernen Gesellschaften - 
wie immer man sie nennen will - ist dies nicht mehr so. Handlungen werden multidimensional in ihren Konsequenzen. Sie können ebenso an ihrem Ausgangspunkt wie räumlich und zeitlich weit davon entfernt zu Veränderungen führen. Auch Alltagswissen bezieht sich nicht mehr nur auf die unmittelbare Umgebung, sondern auf Regionen, die über den ganzen Erdball und noch weiter verstreut sind. Eng mit Globalisierungsprozessen verknüpft ist die Kommunikationsrevolution und die Ausbreitung der Informationstechnologie. Allein der Einfluss des Fernsehens ist beträchtlich, ohne welches die Ereignisse von 1989 in Osteuropa wohl anders verlaufen wären (GIDDENS 1999: 43).

Insgesamt sieht GiDDENS (1999: 46) Globalisierung als ein komplexes Bündel von Prozessen, das durch eine Mischung aus politischen und ökonomischen Faktoren vorangetrieben wird. GIDDENS konzeptualisiert Globalisierung entlang von vier Dimensionen, die eng miteinander verknüpft sind: die kapitalistische Weltökonomie, die internationale Arbeitsteilung, das Nationalstaatensystem und die militärische Weltordnung (GIDDENS 1992). Er hat damit das Feld der Betrachtungen gegenüber der ausschliesslich ökonomischen Argumentation Wallersteins erheblich erweitert.

\subsection{Glokalisierung und der Einbezug von Kultur}

Roland RoberTSON $(1992,1995)$ hat sich bislang wohl am differenziertesten mit der Globalisierung auseinandergesetzt. Die Betonung der kulturellen Dimension teilt er u.a. mit Appadurai (1996), Bauman (1999) und Friedman (1990, 1995). Neben dem fehlenden Einbezug von Kultur kritisiert RoBERTSON (1992) u.a. an GIDDENS (und auch anderen), dass er Globalisierung als Konsequenz der Moderne (vgl. BACKHAUS 1996, 1999) sieht und sie somit implizit als tendenziell homogenisierend betrachtet. ROBERTSON dagegen betont die gleichzeitige Präsenz gegenläufiger Bilder von der Globalisierung, woraus man nicht schliessen könne, ob die Globalisierung zur Vereinheitlichung oder zur Fragmentierung führt. Zentral ist dabei, dass sich Untereinheiten des globalen Systems nurmehr mit Bezug zum umfassenden Ganzen konstituieren können, wobei es kein allgemeingültiges, dominantes Modell gibt, an dem sich die Untereinheiten oder Gesellschaften orientieren können. Jede Gesellschaft kreiert und kommuniziert ihr eigenes, spezifisches Bild einer globalen Ordnung, an der sich z.B. die nationale Identität spiegelt. Durch das Zusammenspiel dieser Bilder wiederum entsteht eine gemeinsame Vorstellung von dem, was als global universal gilt. Deswegen spricht ROBERTSON von der Gleichzeitigkeit der «Partikularisierung des Universalismus»-gewissermassen den lokalen Vorstellungen einer gemeinsamen Welt - und der «Universalisierung des Partikularismus»-z.B. die globalisierte Erwartung, dass Gesellschaften eine spezifische, abgegrenzte Identität bzw. Kultur haben (sollen). Damit hat ROBERTSON auch verdeutlicht, dass es sich bei der Globalisierung nicht um ein eindeutig wahrnehmbares und beschreibbares Phänomen handelt und dass es fortlaufend in den verschiedenen Diskursen neu konstruiert wird.

Um den Zusammenhang homogenisierender, bzw. universalisierender und heterogenisierender, bzw. partikularisierender Tendenzen aufzuzeigen, die unser Leben um das fin de siècle bestimmen, schlägt er den Begriff «Glokalisierung» vor (ROBERTSON 1995). Denn verbunden mit der Frage, ob Globalisierung homogenisierend oder fragmentierend wirke, ist die (vermeintliche) Polarität zwischen Lokalem und Globalem, die GIDDENS als dialektisch begreift, was jedoch nur einen Teil dieses Gegensatzes erklärt (RoBERTSON 1995: 27). Der Begriff Globalisierung hat seinen Ursprung im japanischen Businessjargon der 80er Jahre. Er wird verwendet, wenn Produkte mit globaler Verbreitung für einen lokalen Markt getrimmt werden, sei dies durch Modifikationen (z.B. in der Automobilherstellung) oder durch spezifische Werbung (z.B. bei Softdrinks und Zigaretten). ROBERTSON fasst den Begriff weiter. Er postuliert, dass jeder Prozess der Globalisierung, der als homogenisierend angesehen wird, auch eine fragmentierende, bzw. heterogenisierende Komponente aufweist. So wird z.B. das (homogenisierende) Internet auch dazu verwendet, sezessionistische (fragmentierende) Bewegungen zu verbreiten. Auch die auf den ersten Blick so anti-globale islamische Revolution im Iran konnte nur erfolgreich sein, weil sie sich der Bürokratie des Nationalstaates, der auf einem globalen Konzept beruht, bedienen konnte (BEYER 1994: 160ff). Auch die Berufung auf eine bestimmte, sich abgrenzende kulturelle Identität ist nur durch universale Annahmen darüber, was eine Kultur überhaupt ausmacht, bzw. wodurch sich Kulturen voneinander abgrenzen, möglich.

\section{Globalisierung erfordert eine translokale Konzeption von Kultur}

In Bezug auf Kultur verdeutlicht die Konzeptionalisierung der Globalisierung als Glokalisierung zwei Dinge: Erstens werden wir darauf aufmerksam gemacht, dass die Wahrnehmung eines globalen Mosaiks von unterscheid- und abgrenzbaren Kulturen erst durch global verbreitete Annahmen darüber, dass und wie sich Kulturen voneinander unterscheiden, seine heutige Form bekommen konnte. Zweitens wird klar, dass ein Verständnis, das Kultur als territorial, statisch und aus einem lokalen Lern- und Sozialisationsprozess entstanden sieht, nicht (mehr) den Bedingungen der Globalisierung gerecht werden kann und somit ein anderes Kulturverständnis gefordert ist. Dieses Verständnis von Kultur sollte vermehrt auch in der Geographie berücksichtigt werden. 


\subsection{Problematische statische Konzeption von Kultur}

Gemäss statischem Verständnis wird Kultur als authentisch, beständig, abgegrenzt und einer bestimmten Gruppe oder Gesellschaft eigen bzw. zugehörig gesehen. Bleibt man diesem statischen, auf Authentizität fussenden Konzept verhaftet, läuft man Gefahr, Kultur zusammen mit dem Aufenthaltsort ihrer TrägerInnen zu verorten und territorial zu verstehen. Werden letztere nun mit Elementen anderer, fremder, ferner, exterritorialer Kulturen konfrontiert - wie das durch Prozesse der Globalisierung immer häufiger und intensiver der Fall ist - so stehen sie vor der Frage, ob dies mit der eigenen Kultur verträglich ist, ob es also - mit gewissen Anpassungen - vereinnahmt werden kann, oder ob es als bedrohlich empfunden und abgelehnt werden muss. Ad extremum führt SAMUEL HunTINGTON (1993, zit. nach EHLERS 1996) v.a. die bedrohliche Variante dieses Verständnisses aus, indem er von einem «clash of civilizations» spricht, der als Folge von kulturellen Unterschieden, bzw. Unverträglichkeiten, (bewaffnete) Konflikte zwischen Zivilisationen unausweichlich mache. Dadurch wird Kultur (bzw. Zivilisationen, die auf kulturellen Gemeinsamkeiten beruhen) territorial verortet und diese Verortung wird naturalisiert, also als vorgegeben betrachtet. Wie fatal eine derartige Naturalisierung ist, zeigt sich in vielen Konflikten - z.B. in Kosovo, Nordirland, Tschetschenien, Aceh und Osttimor, um nur einige zu nennen - wo sich die Konfliktparteien auch auf kulturelle Unverträglichkeiten beziehen. Deutlich wird hier aber auch dass Kultur immer konstruiert ist (vgl. HaBermas 1998: 116ff.), was oft vergessen geht. Die Folgen eines naturalisierten Kulturkonzeptes werden damit fatalerweise zur Ursache von Konflikten gemacht.

Auch wenn es nicht zum offenen Konflikt kommt, kann ein statisches Kulturverständnis, gepaart mit einer eindimensionalen Sicht von Globalisierung, wie dies im öffentlichen Diskurs häufig der Fall ist, protektionistische Reaktionen hervorrufen. Diese sind einer Lösung globaler Probleme eher hinderlich, wie die Ausgrenzung von Fremden und Fremdem z.B. zur Bewahrung traditioneller Werte im konservativen Lager oder zur Bewahrung sozialer oder ökologischer Standards auf der Seite linker und grüner Gruppierungen zeigen (BECK 1997, ZÜRN 1998).

\subsection{Translokale Kultur als menschliche Software}

Ein Verständnis von Kultur, das Prozessen der Globalisierung nicht mit Protektionismen begegnet, darf Kultur nicht statisch auffassen und lokal verorten und abgrenzen. Vielmehr sollte Kultur als fliessend und sich translokal entwickelnd begriffen werden. Um es zeitgemässer auszudrücken, müssen wir Kultur nicht als Hardware betrachten, mit der man ausgerüstet wird, sondern als Software, die man sich aneignet, anpasst und durch den Gebrauch stets verändert. FriedmaN
(1990) nähert sich einem solchen Kulturverständnis über den Konsum. Denn mit dem Konsum von Waren und Dienstleistungen konsumieren wir auch Kultur. Nur schon dadurch, was und wie wir essen, identifizieren wir uns mit bestimmten Aspekten von Kultur. Ein Beharren auf Authentizität lässt sich gerade unter Bedingungen der Globalisierung nicht aufrecht erhalten. Die Meinung, dass die (eigene) Kultur nur durch den Konsum spezifischer (eigener) Güter und Dienstleistungen aufrechterhalten werden könne, bzw. durch den Konsum von Fremdem verändert, verwässert oder bedroht werde, ist irreführend und wie oben gezeigt, gefährlich. Vielmehr ist es möglich, gerade durch den Konsum von als fremd Eingestuftem, eine distinkte eigene Kultur zu erhalten, bzw. zu generieren. FrIEDMAN (1990: 314-325) führt in der Folge zwei - auf den ersten Blick kontrastierende - Beispiele an, das der Sapeurs in der Volksrepublik Kongo und der Ainu, einer ethnischen Minderheit in Japan.

Die Sapeurs (die sich nach dem Ausdruck se saper nennen, was soviel wie «sich gut kleiden» bedeutet), gehören eher der unterprivilegierten Gesellschaftsschicht an. Sie versuchen explizit über den Konsum westlicher Kleidung und anderer Konsumgüter, ihren Status zu erhöhen. Es geht dabei jedoch nicht in erster Linie darum, westlichen Idealen nachzueifern, bzw. die westliche Kultur zu übernehmen, sondern vielmehr darum, mit den Mitteln der Konsumption den Status in der lokalen Gemeinschaft zu verbessern. Die statussteigernde Bewertung westlicher Konsumgüter kann wohl auf die Kolonialzeit und die Modernisierung zurückgeführt werden, doch ist sie per se kein globaler, sondern ein lokaler (oder besser glokalisierter) kultureller Aspekt (FRIEDMAN 1990: 314-319).

Die Ainu werden von EthnologInnen als auf der nördlichen Insel Hokkaido lebende Sammler- und Jägerethnie bezeichnet, die ökonomisch und politisch als unterprivilegiert gilt. Ihr Territorium wurde im 19. Jh. dem Meiji-Staat einverleibt. Von den die Mehrheit der JapanerInnen stellenden Nihonjin werden sie allerdings nicht als Ethnie anerkannt, sondern als sozial schlecht eingebundene, unterprivilegierte Schicht, die es besser zu integrieren gelte. Allerdings haben es die Ainu, die sich integrieren wollen, nicht einfach, da sie aufgrund ihrer (sozialen, nicht der kulturellen) Herkunft als «outcasts» stigmatisiert werden. In den 70er Jahren formierte sich eine kulturelle Bewegung, welche die Anerkennung der Ainu als eigenständige Ethnie forderte, was die japanische Gesellschaft, die sich nicht als multikulturell oder multiethnisch versteht, vor nicht zu unterschätzende Probleme stellte. In der Folge richteten die Ainu lokale Schulen ein, bauten traditionelle Häuser wieder auf und stellten traditionelle Handarbeiten her, die touristisch vermarktet werden. Über die Kommodifizierung ihrer Kultur gelang es den Ainu also, sich ihre «traditionelle» Kultur neu zu erschaffen (FrIEDMAN 1990: 320-322). 
Man würde meinen, durch ihre starke Konsumorientierung müssten sowohl die Sapeurs als auch die Ainu kulturellen Selbstmord betreiben, doch genau das Gegenteil ist der Fall: sie kreieren ihre (eigenständige) Kultur neu durch den Konsum globaler Produkte oder die Kommodifizierung lokaler Produkte für den globalen Markt. Aus der Sicht statisch verstandener Kultur, müsste man einräumen, dass sowohl die Kultur der Sapeurs, als auch die der Ainu ihre Authentizität verloren haben, auch wenn sie ihre, bzw. eine kulturelle Eigenständigkeit wahren konnten.

Als Beispiel für eine lang bestehende Kultur, deren TrägerInnen sich auf translokale Weise mit Prozessen der Globalisierung auseinandergesetzt haben, kann die balinesische genannt werden. Die kleine Insel im indonesischen Archipel wurde vor der Asienkrise geradezu von TouristInnen überschwemmt, die einerseits durch günstige Flugpreise, tiefe Lebenshaltungskosten, Strände und Palmen, anderseits aber auch durch die «farbenfrohe Kultur» angezogen wurden (vgl. BACKHAus 1997). Die Anwesenheit von Fremden bei den zahlreichen Ritualen sowie der gänzlich andere Lebensstil der Reisenden, blieb für die balinesische Kultur nicht folgenlos. Allerdings sollte nicht von einem Verlust der Kultur gesprochen werden, auch wenn heute gewisse Rituale anders vollzogen werden und sich das Alltagsleben vieler BalinesInnen durch den Einfluss der Globalisierung verändert hat. Denn das Fremde wurde weder fraglos als gut betrachtet und übernommen, noch gänzlich abgelehnt und ausgegrenzt. Vielmehr wurden die Elemente übernommen und lokalisiert, die als nützlich erschienen (z.B. die Verwendung von Lastwagen, damit mehr Menschen aus einem Dorf zu einem Tempel pilgern können; die Investition von Geldern aus dem Tourismusgeschäft für die «bessere», den Göttern angemessenere Durchführung von Ritualen etc.). Ausserdem werden mehr Diskussionen darüber geführt, was man an anderen sowie der eigenen Kultur wertvoll findet und was nicht. Das ist keineswegs ein einfacher Prozess, doch hat weder die befürchtete Kommodifizierung der Kultur und Degradierung zum Brauchtum stattgefunden (wenn man von einigen problematischen Fällen absieht), noch kam es zur Abschottung und Fundamentalisierung, bzw. zu Protektionismen und Ausgrenzungen.

Im translokalen Kulturverständnis wird also eher nach aussen als nach innen, eher auf Gemeinsamkeiten als auf Trennendes geachtet. Grenzen werden als Übergänge, als Diffusionsmöglichkeit gesehen, nicht als Ende der eigenen, sondern als Beginn einer gemeinsamen Kultur. Dies beinhaltet jedoch nicht, dass Anpassung und Mischung bzw. Hybridisierung (vgl. PIETERSE 1995) kultureller Aspekte zu einer kulturellen Entropie und somit zur Vereinheitlichung führen. Vielmehr wird einem durch die aktive Auseinandersetzung mit Fremdem das Eigene bewusster, das dann aber - und dies macht den wesentlichen Unterschied aus - neu bewertet und reflektiert wird und nicht weil es ein immanenter (tradierter) Wert der eigenen Kultur ist, per se als Wert eingestuft wird.

Man muss sich also überlegen, welche Werte einem wieviel «wert» sind und warum (BAUMAN 1999). Denn die Anerkennung eines Wertes beinhaltet - das wird oft vergessen - auch den Verzicht auf anderes.

\subsection{Translokal (ver)handeln}

Die Berücksichtigung eines fliessenden, translokalen Kulturverständnisses, bei dem man Betroffenheiten auch über den Zaun der eigenen Kultur hinweg sieht und sich damit identifiziert und solidarisiert, ist noch kein Garant für eine Lösung von mit Prozessen der Globalisierung verbundenen Problemen, doch ein erster Schritt dazu. So konnte bspw. das von OECD-Mitgliedstaaten klandestin geplante - u.a. für Entwicklungsländer problematische - MAI (Multilateral Agreement on Investment) nur durch eine konzertierte globale Internetkampagne an die Öffentlichkeit gebracht (und vorläufig) verhindert werden (DE BRIE 1998, WELZK 1999). Auch der Erdgipfel von Rio 1992 und die nachfolgenden Klimakonferenzen können als Schritt in diese Richtung gesehen werden, auch wenn die Umsetzung der Beschlüsse oftmals an Protektionismen scheitern.

Um uns bewusst zu machen, welche (Um-)Welt wir wollen, müssen wir global denken, um unsere (gemeinsamen) Werte zu vertreten, müssen wir translokal handeln. Problematisch dabei ist, dass unter gegenwärtigen Bedingungen die Wirksamkeit politischer Regelungen von der Erfüllung der sog. Kongruenzbedingung abhängt: der Raum, in dem sich gesellschaftliche Austauschbeziehungen und Handlungszusammenhänge verdichtet haben, darf nicht viel grösser sein als der Raum, der durch politische Regelungen erfasst wird (ZüRN 1998: 298-301, HABERMAS 1998: 91 ff.). Dies ist aufgrund globalisierender Prozesse zunehmend weniger der Fall. So kann z.B. der Versuch einer nationalen Notenbank, die Konjunktur durch Erhöhung der Zinssätze abzukühlen, durch eine us-amerikanische Zinssenkung ins Leere laufen. Oder eine Senkung der Hautkrebsrate - die aufgrund einer beschädigten Ozonschicht steigt - kann nicht mehr durch nationale Politik allein erreicht werden. Protektionismen, welcher Couleur auch immer, tragen dabei wenig bis gar nicht zur Lösung dieser Probleme bei. Es wird darum in Zukunft weit häufiger und unter anderen Bedingungen - da neben Staaten zunehmend auch nicht-staatliche international tätige Akteure ins Geschehen eingreifen - verhandelt werden müssen, als dies heute der Fall ist. Wir sind noch weit von einer auf einer Solidarität der WeltbürgerInnen fussenden «kosmopolitischen Demokratie», bzw. einem «postnationalen Bundesstaat» (Habermas 1998: 163-164) entfernt, welche der Bewältigung gegenwärtiger Probleme eher gewachsen wären. Doch kommen wir diesen ein gutes Stück näher, 
wenn wir versuchen, im öffentlichen Diskurs die Aufmerksamkeit auf diese Zusammenhänge zu lenken und damit die Rahmenbedingungen zu schaffen, die eine solche Solidarität fördern. Ein Konzept von Globalisierung, das der kulturellen Dimension Rechnung trägt und ein fliessendes, translokales Kulturverständnis, kann unseres Erachtens dazu einen wichtigen Beitrag leisten.

\section{Literatur}

ApPadurai, A. (1996): Modernity at Large - Cultural Dimensions of Globalization. - Minneapolis/London: University of Minnesota Press.

ARNASON, J.P. (1990): Nationalism, Globalization and Modernity. - In: Featherstone, M. (eds.): Global Culture - Nationalism, Globalization and Modernity. London, Sage: 207-236.

BACKHAUS, N. (1996): Globalisierung, Entwicklung und traditionelle Gesellschaft - Chancen und Einschränkungen bei der Nutzung von Meeresressourcen auf Bali/Indonesien. - Münster: Lit.

BackHaus, N. (1997): Tourismus auf Bali - vom Paradies zum Museum. - In: Tourismus Journal 1/1: 90-116. Backhaus, N. (1999): Zugänge zur Globalisierung, Konzepte, Prozesse, Visionen. $-=$ Anthropogeographie 17, Zürich.

Bauman, Z. (1999): Culture as Praxis. - London: Sage. BECK, U. (1997): Was ist Globalisierung? - Frankfurt am Main: Suhrkamp.

BEYER, P. (1994): Religion and Globalization. - London: Sage.

BRIE DE, CH. (1998): Wie das MAI zu Fall gebracht wurde - Die Globalisierung des Widerstands. - In: Le Monde diplomatique 12/1998: 7.

DiCKEN, P. (1998): Global Shift - Transforming the World Economy. - London: Paul Chapman.

EhLeRS, E. (1996): Kulturkreise - Kulturerdteile Clash of Civilizations: Plädoyer für eine gegenwartsbezogene Kulturgeographie. - In: Geographische Rundschau 48/6: 338-344.

Featherstone, M., Lash, S. \& Robertson, R. (eds.) (1995): Global Modernities. - London: Sage.

FRIEDMAN, J. (1990): Being in the World: Globalization and Localization. - In: Featherstone, M. (ed.): Global Culture - Nationalism, Globalization and Modernity. - London, Sage: 311-328.

Friedman, J. (1995): Global System, Globalization and the Parameters of Modernity. - In: Featherstone, M., LASH, S. \& Robertson, R. (eds.): Global Modernities. - London, Sage: 69-90.

GidDENS, A. (1992): The Consequences of Modernity. - Cambridge: Polity Press.

GidDENs, A. (1995): Die Konstitution der Gesellschaft - Grundzüge einer Theorie der Strukturierung. - Frankfurt/New York: Campus.
Giddens, A. (1999): Der dritte Weg - Die Erneuerung der sozialen Demokratie. - Frankfurt am Main: Suhrkamp.

Gollmer, M. (1999): Grenzen nationaler Politik. - In: Tages-Anzeiger der Stadt Zürich, 13. März: 7.

HABERMAS, J. (1998): Die postnationale Konstellation Politische Essays. - Frankfurt am Main: Suhrkamp.

HofFMANN, M. (1999): Globalisierung im öffentlichen Diskurs. - Diplomarbeit, Universität Zürich, Zürich.

Panajotis, K. (1996): Globalisierung, Politik, Verteilung - Arbeitsgesellschaft im Umbruch (V): Die grosse Seligkeit wird nicht kommen. - In: Tages-Anzeiger der Stadt Zürich 29. November: 89.

Perraton, J., Goldblatt, D., Held, D. et al. (1998): Die Globalisierung der Wirtschaft. - In: BECK, U. (Hrsg.): Politik der Globalisierung. - Frankfurt am Main, Suhrkamp: 134-168.

Pieterse, N. (1995): Globalization as Hybridization. In: Featherstone, M., Lash, S. \& Robertson, R. (eds.): Global Modernities. - London, Sage: 45-68.

RoberTson, R. (1992): Globalization - Social Theory and Global Culture. - London: Sage.

RoBERTSON, R. (1995): Glocalization: Time-Space and Homogeneity-Heterogeneity. - In: FEATHERSTONE, M., LASH, S. \& Robertson, R. (eds.): Global Modernities. - London, Sage: 25-44.

ScHMidT, H. (1998): Globalisierung - Politische, ökonomische und kulturelle Herausforderungen. - Stuttgart: Deutsche Verlagsanstalt.

SPIELER, M. (1998): Milliardeneinbusse für die UBS. In: Tages-Anzeiger, 25. September: 1; 35.

WaGNer, P. (1996): Viel Dunst umweht die Positionen - Die Arbeitsgesellschaft im Umbruch (I): Globalisierung als Ideologie und Erfahrung. - In: TagesAnzeiger, 26./27. Oktober: 57.

Wallerstein, I. (1990): Culture as the Ideological Battleground of the Modern World System. - In: FEATHERSTONE, M. (eds.): Global Culture - Nationalism, Globalization and Modernity. - London, Sage: 31-56.

WelzK, S. (1999): Visionen des MAI - A Brave New Corporatist World? - In: Blätter für deutsche und internationale Politik 44/1: 40-50.

ZüRN, M. (1998): Schwarz-Rot-Grün-Braun: Reaktionsweisen auf Denationalisierung. - In: BECK, U. (Hrsg.): Politik der Globalisierung. - Frankfurt am Main, Suhrkamp: 297-330.

\section{Zusammenfassung: Globalisierung und translokales Kulturverständnis}

Im Kontext der Globalisierungsdiskussion wird ein Kulturverständnis thematisiert, das Kultur nicht statisch, sondern als räumlich und zeitlich dynamisch betrachtet. Eine einseitige Wahrnehmung der Globalisierung als homogenisierend und wirtschaftszentriert wird - gepaart mit einem statischen, auf Authentizität ausge- 
richteten Kulturverständnis - häufig als Bedrohung empfunden und führt zu protektionistischen Reaktionen, bzw. zur Ablehnung der Globalisierung. In diesem Artikel werden also Konzepte von Kultur und Globalisierung im wissenschaftlichen und medialen Diskurs sowie deren Implikationen und Konsequenzen diskutiert. Im medialen Diskurs wird Globalisierung häufig als etwas Unausweichliches thematisiert, deren Folgen - z.B. Arbeitsplatzvernichtung, Klimaveränderung oder kulturelle Vereinheitlichung - wir ausgeliefert sind. Im weiter gefächerten wissenschaftlichen Diskurs wird Globalisierung differenzierter betrachtet. Im vertretenen Ansatz wird argumentiert, dass Lokales und Globales nicht Gegensätze, sondern sich bedingende Elemente des Globalisierungsprozesses sind. Daraus wird geschlossen, dass ein translokales Kulturverständnis weit besser zur Lösung von globalen Problemen beitragen kann, als ein statisches.

\section{Summary: Globalization and Translocal Culture}

In the context of the debate about globalization a concept of culture is discussed that regards culture not as static but as dynamic over space and time. A static concept of culture that is oriented to authenticity, connected to a one-sided notion of globalization as a homogenizing and mostly economic process, is often perceived as a threat. As a consequence, this often leads to protectionist reactions and to the rejection of everything that is connected with the word globalization. Different concepts of culture and globalization and their implications and consequences will be discussed as they are used in the scientific and media discourse. In the media discourse globalization is often portrayed as something we cannot escape from and of which we only have to bear the consequences - i.e. job cuts, climatic changes or cultural standardisation - without being able to do something about it. In the broader scientific discourse we encounter more complex and differentiated notions about globalization. Using a theory that does not regard the local as antagonistic to the global but as mutually dependent elements of the globalization process, it is concluded that - compared with a static concept of culture - a translocal concept is much more useful for the solution of global problems.

\section{Résumé : Mondialisation et culture translocale}

Dans le cadre du débat sur la mondialisation, on conçoit une notion de la culture qui est dynamique dans l'éspace et dans le temps et non pas statique. La perception, étroite, d'une mondialisation homo-généisante et centrée sur l'économie - associée à la notion d'une culture qui soit statique et en quête d'authenticité, est souvent ressentie comme une menace et provoque des réactions de protectionisme, de refus de mondialisation. Dans cet article, il est question des conceptions de la culture et de la mondialisation, ainsi que de leurs implications et conséquences dans le discours scientifique et le discours médiatique. Dans le discours médiatique, la mondialisation est souvent présentée comme une fatalité dont les conséquences, par exemple réduction d'emplois, rechauffement climatique et uniformisation culturelle. Le discours scientifique présente une idée plus nuancée de la mondialisation. L'article estime que «le local» et «le global» ne sont pas contradictoires, mais inter-dépendants, parties intégrales du processus de mondialisation. On conclut qu'une conception translocal de la culture contribue à résoudre les problèmes globaux avec plus d'éfficacité qu'un concept plus statique.

Dr. phil. Norman Backhaus, Dipl.-Geogr. Marco Hoffmann, Geographisches Institut der Universität Zürich, Winterthurerstr. 190, CH-8057 Zürich.

e-mail: backhaus@geo.unizh.ch 